\title{
EDITORIAL
}

\section{Can three-dimensional imaging provide insights into spatial variations in bacterial infection}

patterns?

Peter W. Taylor ${ }^{1 *} \&$ Kevin P. Francis ${ }^{2}$

${ }^{1}$ School of Pharmacy, University College London, London WC1N 1AX, United Kingdom

${ }^{2}$ PerkinElmer, Hopkinton, MA 01748, United States of America

Author correspondence: Tel.: +44 207753 5867; peter.taylor@ucl.ac.uk

Keywords: Three dimensional imaging; computed tomography; bioluminescence imaging; bacterial infection; animal models of infection; Escherichia coli; Citrobacter rodentium; DLIT- $\mu$ CT 
Abstract: Recently developed three-dimensional (3D) non-invasive in vivo optical imaging is providing fresh insights into the understanding of the pathogenesis of invasive bacteria in small animal experimental models. Here, we describe the advantages of 3D diffuse light imaging tomography with integrated micro-computed tomography (DLIT- $\mu \mathrm{CT}$ ) over more traditional 2D systems, in particular with regard to precise localisation of infectious foci within tissues in threedimensional space. We highlight data from rodent studies that employ experimental infections replicating the course of naturally occurring bacterial disease, such as invasive Escherichia coli infections that arise following colonisation of the gastrointestinal tract in neonatal rats. It is argued that this technology will find increasing utility in the study and diagnosis of infectious disease.

\section{Text}

A deeper understanding of the temporal and spatial dynamics of localised and disseminated bacterial infections will provide enormous benefit for preclinical research, especially with regard to the comprehension of mechanisms of bacterial pathogenicity and for the evaluation of badly-needed novel therapies. Moreover, this information will prove invaluable for clinical translation and provide greater insight for precise diagnosis and monitoring of human and animal infectious disease. Rapid advances in tomographic non-invasive imaging techniques, such as magnetic resonance imaging (MRI), computed tomography (CT), single photon emission computed tomography (SPECT) and positron emission tomography (PET), together with recently developed three-dimensional optical imaging approaches, provide researchers with the opportunity to monitor molecular and anatomical changes associated with disease progression and therapeutic intervention, particularly when two or more of these imaging modalities are used in combination [1]. In comparison to other disease areas, in particular oncology, clinical use of these imaging technologies is in its infancy, in large part due to the inability of current procedures to distinguish sites of bacterial infection from sites of sterile inflammation, wound healing and the like. However, recent development of several tracers that specifically target bacteria in vivo may help to overcome these limitations; these include a number of 
cell wall binding antibodies, antibiotics and antimicrobial peptides, as well as novel probe chemistries such as membrane-interactive zinc(II)-dipicolylamine [2,3].

Both 2D and 3D real time optical imaging have made a significant contribution to our understanding of bacterial pathogenesis in small animal models of infection. Many of these advances have been facilitated by the employment of fluorescent and bioluminescent genetically encoded reporters (i.e., fluorescent proteins and luciferases) for the labelling of pathogens of interest. Use of these optically labelled bacteria in conjunction with a new generation of instruments that merge functional optical imaging with anatomical imaging modalities, including $\mathrm{CT}$, has enabled a better understanding of the dynamics of these microbial diseases [4,5]. Although whole animal bioluminescence imaging has in the past been somewhat restricted by the availability of only a limited number of reporter systems such as the lux operon (IuxCDABE) from the terrestrial bacterium Photorhabdus luminescens or Firefly luciferase, an ever increasing number of luciferases have been shown to work effectively in different bacterial strains, including Gaussia, Renilla, Click Beetle and, most recently, NanoLuc luciferase. Moreover, these bioluminescent reporters have significant advantages over biofluorescent reporters, with better light penetration yielding a lower background, greater sensitivity, high specificity and the capacity for engineering into a wide variety of bacteria [6]. Imaging is most relevant when the reporter is maintained in stable fashion on the bacterial chromosome and does not disrupt pathogenic potential by insertion into and subsequent disruption of key virulence genes; to achieve this may require screening of a large number of conjugants [7]. The full potential of these constructs can be realised in combination with multimodality imagers such as the IVIS Spectrum-CT system, which combine 3D diffuse light imaging tomography with integrated $\mu \mathrm{CT}$ (DLIT- $\mu \mathrm{CT}$ ) to obtain accurate non-invasive anatomical localisation of bioluminescent bacterial foci in vivo without the need for ex vivo analysis of excised organs and tissues [8]. Most importantly, animal modellers should attempt to replicate, as much as possible, the natural history of the bacterial infection as it occurs in the natural host. All too often the physiological and anatomical barriers to infection are bypassed by injection of a bacterial bolus 
directly into the blood circulation of an unsuitable animal species, creating an artificial pathogenesis that does not take into account natural processes of dissemination from the site of colonisation.

Animal models which utilise an appropriate animal species and employ an inoculum comparable to the natural infectious dose range, delivered in analogous fashion to the natural route of infection, should provide reliable tools with which to image the infectious process from colonisation to systemic or mucosal endpoint. For example, we have developed a model of Escherichia coli K1 infection in two-day-old rats that to a large extent recapitulates the infection in the human neonate [9]. The mouse is unsuitable as a model for this neonatal infection as susceptibility to infection is not strongly age-related in this species. Oral administration of a small number of bacteria leads to colonisation of the small intestine, expansion of the colonising population on the mucosal surface, translocation by an intracellular pathway to the sub-epithelium, passage through the mesenteric lymphatic system and transit through the blood and choroid plexus to colonise and initiate inflammation of the meninges [9], creating an age-dependent infection with many similarities to the human condition and facilitating real-time imaging in fine detail [7].

2D imaging has been used extensively to evaluate disease status in small animals and to progress novel therapeutics towards clinical evaluation [10]; studies include modelling of a range of Gram-positive and Gram-negative bacterial infections. In comparison, 3D imaging using DLIT- $\mu C T$ has not been widely used: the technology is relatively new, the equipment is more expensive and labour requirements are somewhat more intensive than those needed for $2 \mathrm{D}$ imaging systems. It does, however, provide a number of advantages: for 2D bioluminescence imaging, the optical camera collects light only from the surface of the animal and therefore does not localise the signal within a three-dimensional space. The positioning of the animal influences $2 \mathrm{D}$ image quality and intensity and may lead to reproducibility issues in longitudinal studies. New generation DLIT- $\mu C T$ imagers incorporate CT scanning for 3D signal co-registration for simultaneous provision of molecular and anatomical images, enabling precise location of signals in three-dimensional space, assembly of images that can be rotated and generation of 4D movies of disease progression and 
infection cycles. Examples may be found in the Supplemental Material from reference [7] and on the BBC website site - http://www.bbc.com/news/health-18631157. It is usually necessary to validate the location of $2 \mathrm{D}$ signals at post-mortem by tissue excision and imaging of tissue sections whereas this is not normally necessary if 3D optical tomographic methods are applied, enabling real time longitudinal studies in single animals. Both 2D and 3D bioluminescent imaging protocols are likely to reduce the number of animals employed for evaluation of disease burden, particularly welcome in high throughput drug screening programmes. Due to the attenuation of visible light through tissue, especially at wavelengths below $650 \mathrm{~nm}$, it is difficult to detect a bioluminescent signal much beyond a centimetre or two in live animals. For this reason, noninvasive optical imaging is ideally suited for monitoring and tracking disease processes in small animals, such as mice and rats, with only limited utility is larger animals, such as when dealing with superficial disease close to the surface of the animal.

Crucial insights provided by 3D imaging of disseminated bacterial infection are illustrated in Figure 1. In this experiment, twenty adult mice received a bioluminescent uropathogenic strain of $E$. coli (EPEC) by installation into the bladder; the invasive pathogen subsequently ascended the ureters to the kidneys to cause pyelonephritis. When assessed by conventional 2D imaging approximately half the animals showed a unilateral or bilateral kidney signal after 13 days and nearly all animals showed a signal after 20 days. However, careful analysis of 3D images indicated that true pyelonephritis occurred at 13 days, whereas the animals that showed a signal only at 20 days had acquired a gastrointestinal colony (and possibly an infection) of bioluminescent bacteria, in all likelihood due to consumption of contaminated food or faeces. Importantly, the majority of the mice that acquired the gastrointestinal bacteria died, whereas all mice with upper urinary tract infections recovered after antibiotic therapy. Translated to the clinic, a misinterpretation of bacterial distribution such as this could have severe consequences.

A number of recent studies have determined bacterial disease progression in robust small animal models of infection using powerful combinations of 2D and 3D bioluminescent imaging. 
Enteropathogenic E. coli (EPEC) strains are a leading cause of infantile diarrhoea, particularly in developing countries. EPEC is a predominantly human pathogen and is poorly pathogenic in rodents and other small animals [11]. However, disease progression can be investigated in mice with the closely-related murine pathogen Citrobacter rodentium which mimics the course of EPEC human infections $[12,13]$, causing self-limiting colitis. Optical imaging has been used to track the progress of an oral bolus of bioluminescent $C$. rodentium [8]. The bacteria initially colonise the caecum before disseminating to the distal colon; the infection peaks between days 6-8 and resolves 3-4 weeks after initial colonization [14], manifesting as a local increase in bioluminescent signal in the abdomen. 3D multimodality imaging has been particularly useful in establishing that prophylaxis with probiotic bifidobacteria can improve the outcome of $C$. rodentium (and by implication EPEC) infection [14], evaluation of antibiotic treatment and, in conjunction with postmortem evaluations, the host immune response to the gastrointestinal infection [15].

Multimodality imaging seems particularly well suited to infections that follow gastrointestinal colonization. DLIT- $\mu$ CT revealed novel aspects of invasive $E$. coli K1 neonatal infection in the newborn rat that were not apparent using more traditional methods. We identified novel sites of colonization within the alimentary canal, including tongue, oesophagus and stomach, which could contribute to infection severity in the human condition [7]. We also provided further evidence for minimal penetration of brain tissue by the pathogen with colonization restricted to the meninges, a point of contention with regard to E. coli K1 neonatal pathogenesis [16]. In another gastrointestinal study, Kasper and coworkers used 2D and 3D optical imaging to successfully track dissemination from the gut of the anaerobe Bacteroides fragilis and other commensals in a murine model of acute peritonitis to provide real-time analysis of a complex series of host-microbe interactions during the invasive phase of systemic infection [17]. Combinations of 2D and 3D optical imaging have also proven useful in monitoring disease progression in murine models of Mycobacterium tuberculosis [18] and Staphylococcus aureus [19] infections. A wide range of bacterial pathogens have been bioluminescently engineered over the past two decades, including a 
number of BSL3 pathogens such as Mycobacterium tuberculosis, Bacillus anthracis and Yersinia pestis. This has made the study of these diseases much easier and safer, especially in vivo, since the researcher is able to monitor disease progression without unnecessary exposure to the pathogen due to frequent sacrifice of large groups of infected animals and by implementation of laborious microbiology evaluation and enumeration techniques such as CFU plating.

Conclusions DLIT- $\mu C T$ is one of only a few multimodality imaging methods (that include PET and SPECT) with the capacity for true non-invasive localisation of bioluminescent foci in vivo [8]. The small number of studies described above testify to the power of the technology and as 3D instruments become more widely available it is highly likely that they will find increased utility in the study of infectious disease.

Future perspectives As 3D imaging technology becomes mainstream, we anticipate that systems such as DLIT- $\mu$ CT will become widely used as tools to facilitate deeper understanding of spatial and temporal aspects of experimental infections in small laboratory animals. We anticipate that more powerful instrumentation will enable the precise localisation of infectious foci in larger animals, increasing their attraction for the evaluation of antimicrobial chemotherapeutics at all stages of the commercial preclinical development pipeline. Increasing use of efficient extrinsic bioluminescent and fluorescent probes should lead to the realisation of these systems for imaging of naturally arising infections in human subjects.

Financial disclosure: KPF is an employee of PerkinElmer, Inc., manufacturer of optical imaging equipment. 


\section{References}

1. van Oosten $\mathrm{M}$, Hahn M, Crane LM et al. Targeted imaging of bacterial infections: advances, hurdles and hopes. FEMS Microbiol. Rev. 39(6), 892-916 (2015).

2. Bunschoten A, Welling MM, Termaat MF, Sathekge M, van Leeuwen FW. Development and prospects of dedicated tracers for the molecular imaging of bacterial infections. Bioconjug. Chem. 24(12), 1971-1989 (2013). ** developing molecular imaging tracers for optical and nuclear identification of bacteria and bacterial infections

3. van Oosten M, Schäfer T, Gazendam JA et al. Real-time in vivo imaging of invasive- and biomaterial-associated bacterial infections using fluorescently labelled vancomycin. Nat. Commun. 4, 2584 (2013).

4. Close DM, Xu T, Sayler GS, Ripp S. In vivo bioluminescent imaging (BLI): noninvasive visualization and interrogation of biological processes in living animals. Sensors (Basel) 11(1), 180-206 (2011).

5. Witcomb LA, Czupryna J, Francis KP, Frankel G, Taylor PW. Non-invasive three-dimensional imaging of Escherichia coli K1 infection using diffuse light imaging tomography combined with micro-computed tomography. Methods 127, 62-68 (2017).

6. Doyle TC, Burns SM, Contag $\mathrm{CH}$. In vivo bioluminescence imaging for integrated studies of infection. Cell. Microbiol. 6(4), 303-317 (2004).

7. Witcomb LA, Collins JW, McCarthy AJ, Frankel G, Taylor PW. Bioluminescent imaging reveals novel patterns of colonization and invasion in systemic Escherichia coli K1 experimental infection in the neonatal rat. Infect. Immun. 83(12), 4528-4540 (2015). *shows how a combination of 2D and 3D imaging can shed light on an established infection model

8. Collins JW, Meganck JA, Kuo C, Francis KP, Frankel G. 4D multimodality imaging of Citrobacter rodentium infections in mice. J. Vis. Exp. 78, e50450 (2013). **a users' guide to creation of a 4D movie of $C$. rodentium infection 
9. Birchenough GMH, Dalgakiran F, Witcomb LA et al. Postnatal development of the small intestinal mucosa drives age-dependent regio-selective susceptibility to Escherichia coli K1 infection. Sci. Rep. 7, 83, doi:10.1038/s41598-017-00123-w (2017).

10. Byrne WL, DeLille A, Kuo C et al. Use of optical imaging to progress novel therapeutics to the clinic. J. Control. Release 172(2), 523-34 (2013).

11. Croxen MA, Law RJ, Scholz R et al. Recent advances in understanding enteric pathogenic Escherichia coli. Clin. Microbiol. Rev. 26(4), 822-880 (2013).

12. Collins JW, Keeney KM, Crepin VF et al. Citrobacter rodentium: infection, inflammation and the microbiota. Nat. Rev. Microbiol. 12(9), 612-623 (2014). * comprehensive review of the deregulation of intestinal inflammatory responses during induced colitis and role of intestinal microbiota in resistance to colonization

13. Crepin VF, Collins JW, Habibzay M, Frankel G. Citrobacter rodentium mouse model of bacterial infection. Nat. Protoc. 11(10), 1851-1876 (2016).

14. Collins JW, Akin AR, Kosta A et al. Pre-treatment with Bifidobacterium breve UCC2003 modulates Citrobacter rodentium-induced colonic inflammation and organ specificity. Microbiology 158(11), 2826-2834 (2012).

15. Ale A, Crepin VF, Collins JW et al. Model of host-pathogen interaction dynamics links in vivo optical imaging and immune responses. Infect. Immun. 85(1), e00606-16 (2017).

16. Zelmer A, Bowen M, Jokilammi A et al. Differential expression of the polysialyl capsule during blood-to-brain transit of neuropathogenic Escherichia coli K1. Microbiology 154(8), 2522-2532 (2008).

17. Geva-Zatorsky N, Alvarez D, Hudak JE et al. In vivo imaging and tracking of host-microbiota interactions via metabolic labeling of gut anaerobic bacteria. Nat. Med. 21(9), 1091-1100 (2015). *generation of fluorescent anaerobic gut bacteria for imaging of complex bacterial populations in situ 
18. Berod L, Stüve P, Swallow M, et al. MyD88 signalling in myeloid cells is sufficient to prevent chronic mycobacterial infection. Eur. J. Immunol. 44(5), 1399-1409 (2014).

19. Corrado A, Donato P, Maccari S et al. Staphylococcus aureus-dependent septic arthritis in murine knee joints: local immune response and beneficial effects of vaccination. Sci. Rep. 6, 38043. doi: 10.1038/srep38043 (2016). *combination of 2D and 3D bioluminescent imaging deepens understanding of staphylococcal joint infections 


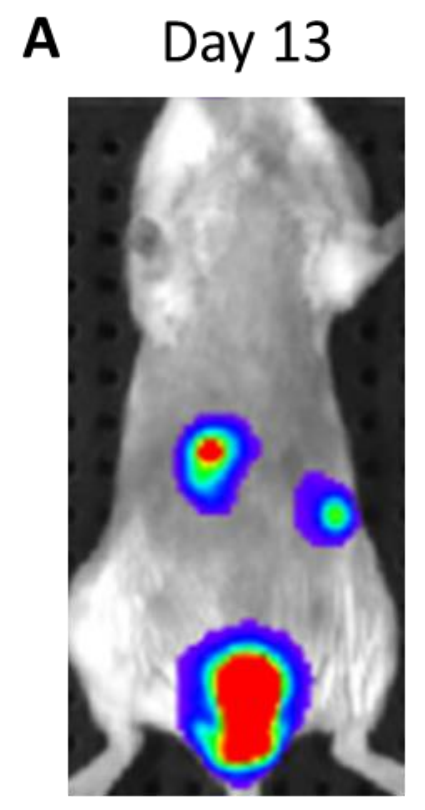

Day 20

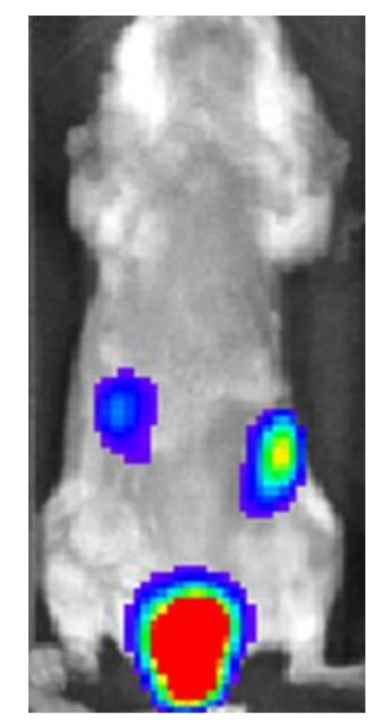

B
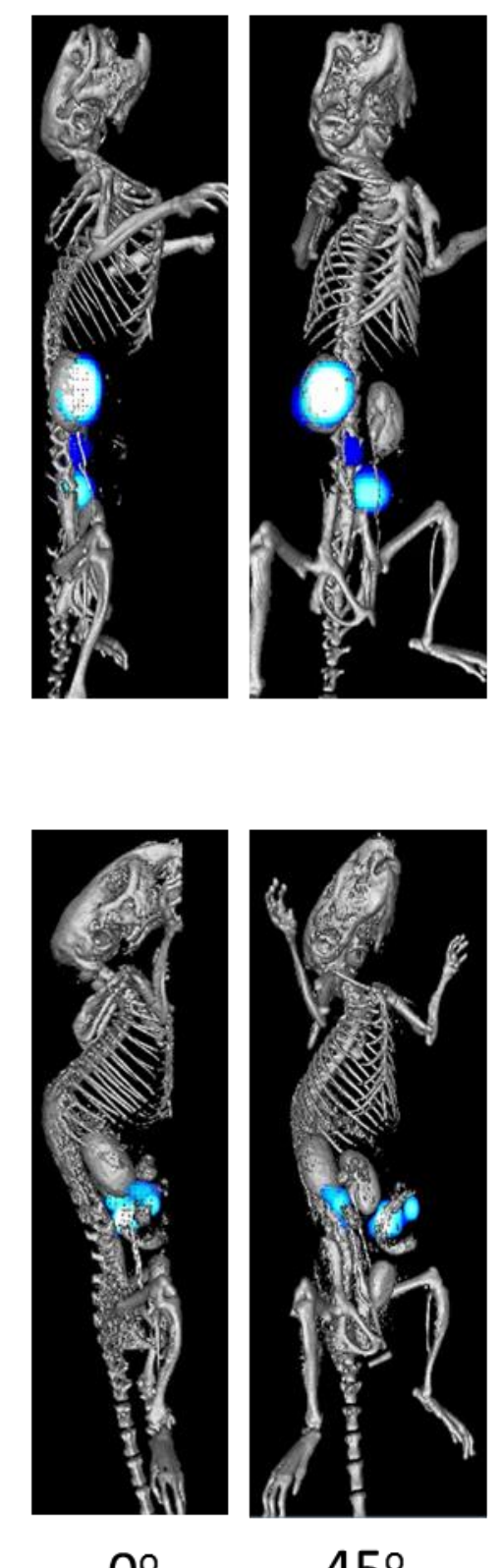

$0^{\circ}$

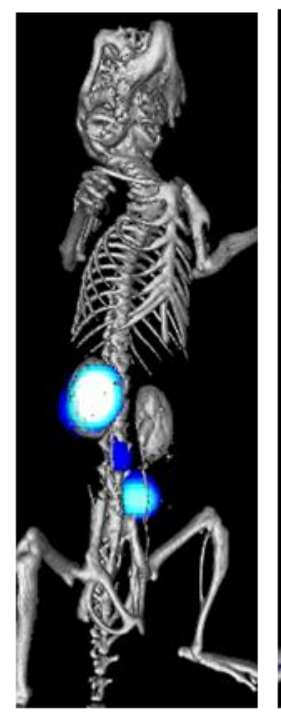

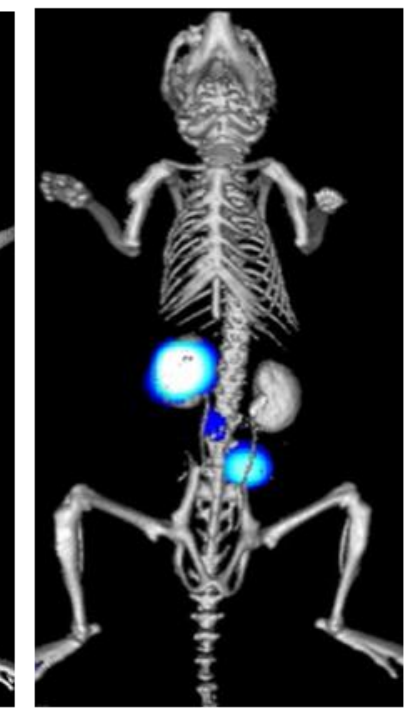

Day 20

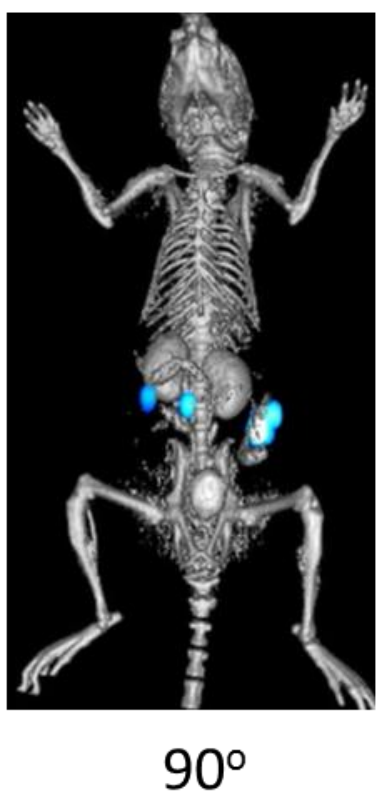

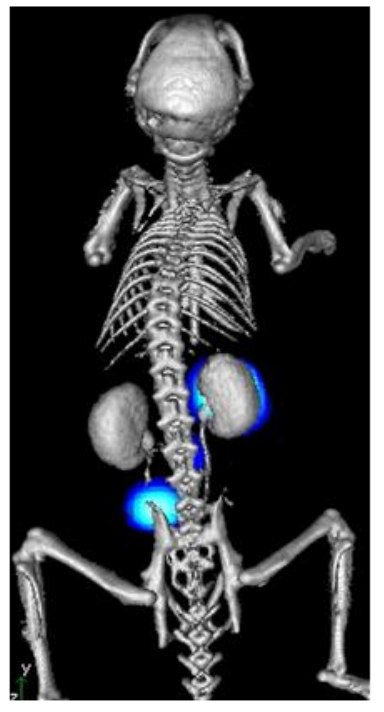

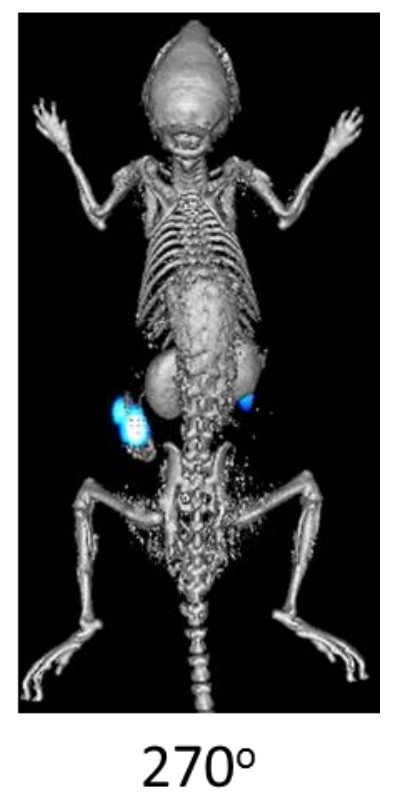




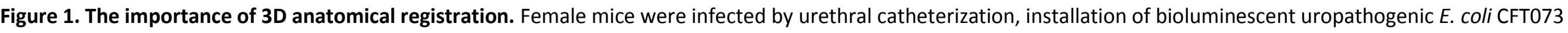

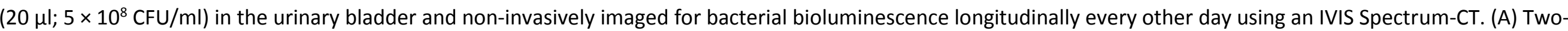

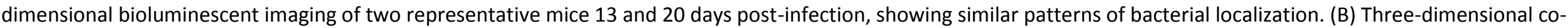

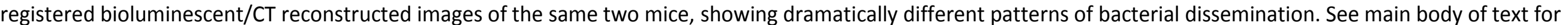
further explanation. 